\title{
Emerging chromatin structural roles of the methyl-CpG binding protein MeCP2
}

\author{
Yui Imaizumi ${ }^{1,2}$ \& Robert Feil*,1,2 (D) \\ ${ }^{1}$ Institute of Molecular Genetics of Montpellier (IGMM), Centre National de Recherche Scientifique (CNRS), 1919 route de Mende, \\ 34293 Montpellier, France \\ ${ }^{2}$ University of Montpellier, 163 rue Auguste Broussonnet, 34090 Montpellier, France \\ *Author for correspondence: robert.feil@igmm.cnrs.fr
}

'Irrespective of the precise underlying mechanisms, the novel studies clearly establish a role for $\mathrm{MeCP} 2$ in the architecture and compaction of heterochromatin, and reveal likely cooperation with HP1 proteins and major-satellite RNAs."

First draft submitted: 3 February 2021; Accepted for publication: 14 February 2021; Published online: 9 March 2021

Keywords: DNA methylation $\bullet$ heterochromatin $\bullet$ MBD proteins $\bullet$ MeCP2 $\bullet$ protein condensates

DNA methylation is an essential epigenetic modification, present in mammals predominantly at cytosine residues that are followed by guanines. In somatic cells, this so-called CpG methylation is found at high levels all along the genome. Different readers have evolved that bind to the methylated DNA and influence chromatin and gene expression. Methyl-CpG binding domain (MBD) proteins constitute the largest family of such proteins, and share a conserved MBD [1]. Extensive research has shown that their binding patterns evolved to facilitate appropriate gene expression levels in the context of highly methylated genomes [2].

Exciting recent studies on the best-studied MBD protein, MeCP2, evoke a new role besides transcriptional repression. They show that $\mathrm{MeCP} 2$ contributes to the $3 \mathrm{D}$ structuration and compaction of heterochromatin as well [3-6]. Below, we present these newly emerging insights and discuss their possible relevance for neurological disorders in humans that are caused by genetic mutations in the $M e C P 2$ gene.

The $\mathrm{MeCP} 2$ gene is positioned on the $\mathrm{X}$ chromosome and is mutated in almost all patients with Rett syndrome (RTT, OMIM 312750), a progressive and devastating autism-spectrum disorder that affects about one in 1015,000 female births [7,8]. Another genetic neurological syndrome is caused by MeCP2 duplication, and is found mostly in males [8]. Constitutive and brain-specific knockout $(\mathrm{KO})$ studies reproduced the clinical phenotype of RTT in mice, and highlighted the essential role of Mecp2 in brain functions [7,9]. Although MeCP2-deficient neurons are functionally abnormal, they do not die. Remarkably, it was shown that the defective brain functions can be largely reversed by reactivating the Mecp 2 gene in the KO mice [10,11]. Thus, although RTT affects neuronal functions, it is not a developmental syndrome.

Mecp 2 produces two similar isoforms that both comprise the MBD but differ slightly in the N-terminus. In the brain, this very highly expressed protein binds not only methyl-CpG but also the methyl-CAC tri-nucleotide motif, which is globally rare in the genome, but more abundant in this than other tissues [12,13]. Extensive research has shown that the protein's primary role is to recruit different proteins involved in transcriptional repression. Among these are the HDAC-containing NCoR1/2 and SIN3A complexes [7]. The recruitment of these repressive complexes by $\mathrm{MeCP} 2$ requires the NCoR1/2 interaction domain (NID) (Figure 1) [7]. Particularly in neurons - where its expression is highest - MeCP2 influences the transcription of many genes in a subtle manner, leading to moderate gains and losses of expression. At genes that show preferential MeCP2 recruitment, its depletion enhances gene expression, indicating that MeCP2's primary role is to dampen gene expression [7]. It had been suggested that MeCP2-binding to genes could also control RNA splicing but this is not supported by recent studies, which also do not provide evidence for a prominent role of methylation in alternative splicing [14]. Besides the MDB and NID, other parts of MeCP2 are also highly conserved and thus could also be functionally important.

Future Medicine 


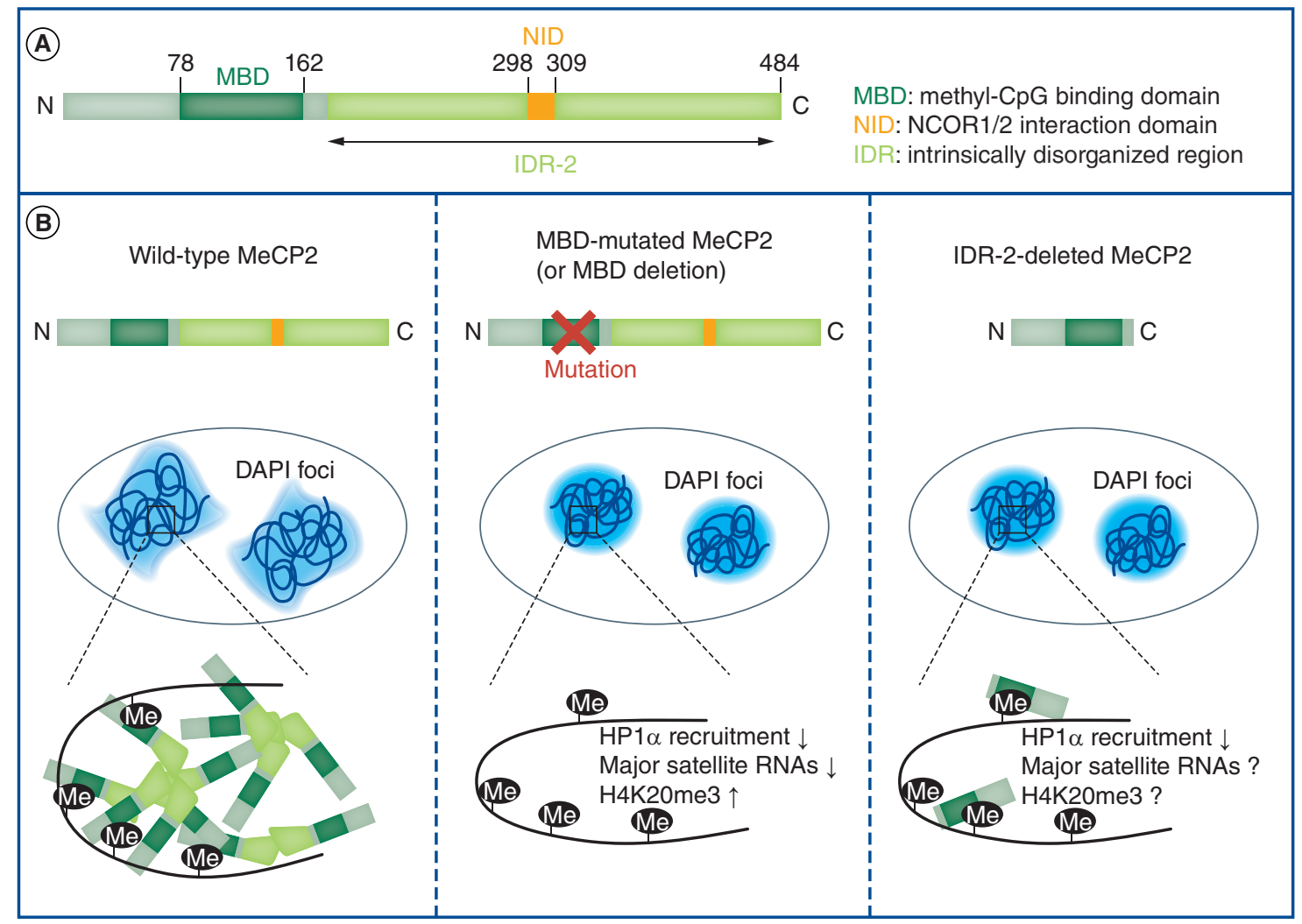

Figure 1. Different domains within MeCP2 contribute to neuronal heterochromatin compaction.

(A) MeCP2 with its functional domains. Shown is mouse MeCP2-B, the most abundant isoform in brain. (B) A model of the chromatin structural effects of deletion/mutation of the MBD domain or removal of the IDR-2 region. Depicted are nuclei (blue ovals) with DAPI-staining heterochromatin. In WT neurons, MeCP2 condensates are present within these foci and this correlates with limited compaction levels. In MBD- and IDR-2 mutant cells, heterochromatin foci are more spherical than in WT cells, due to a lack/reduction of focal MeCP2 condensation. This correlates with reduced levels of HP1 $\alpha$ and major-satellite RNA, and an increase in H4K20me3 [3-6]. IDR: Intrinsically disorganized region; MBD: methyl-CpG binding domain; WT: Wild-type.

Pericentromeric heterochromatin comprises satellite repeat DNA and is particularly rich in methyl-CpG dinucleotides. Immunofluorescence staining on fixed mouse cells shows a marked focal accumulation of MeCP2 in heterochromatic foci. What could be the role of $\mathrm{MeCP} 2$ at these gene-poor heterochromatic domains? To address this question, Linhoff et al. developed ChromAtin, a quantitative approach that combines IF staining and lowtemperature DNA FISH with a method that defines nuclear compartments using array tomography imaging [4]. They carefully compared normal and MeCP2-deficient neurons. Remarkably, the lack of MeCP2 increased DAPI staining at chromocenters, indicating higher compaction levels within pericentromeric heterochromatin. MeCP2 depletion also correlated with increased IF staining of histone H4 lysine-20 tri-methylation (H4K20me3) [4], a modification that was known to control chromatin compaction [15].

Following the discovery that MeCP2 influences chromatin compaction, several groups set out to perform further studies. Applying high-resolution confocal and electron microscopy, Ito-Ishida et al. explored heterochromatic foci of neurons in Mecp2 KO versus WT mice [3]. In hemizygous Mecp $2^{+/-}$females, about half of the neurons do not express the WT Mecp2 allele, due to X-inactivation (Mecp2 maps to the X-chromosome). In these mice, the $\mathrm{MeCP} 2$-deficient neurons showed a markedly stronger DAPI staining of constitutive heterochromatic than the $\mathrm{MeCP} 2$-expressing neurons. MeCP2-depleted heterochromatin foci were more spherical in shape as well, providing another indication that $\mathrm{MeCP} 2$ 'loosens' heterochromatin. Concordantly, when the authors overexpressed MeCP2 in neurons, heterochromatin foci showed reduced DAPI staining and were no longer spherical in shape.

Another remarkable recent study, by Li et al., reported that MeCP2 has physico-chemical properties compatible with the induction of liquid-liquid phase separation (LLPS) condensates in vitro, and that that this propensity of the protein to form droplets is enhanced by the presence of methylated DNA [6]. By studying truncated forms of 
the protein, they discovered that the formation of condensates required a large intrinsically disorganized region (IDR) covering the C-terminal portion of the protein. Generating amino-acid alterations, the authors showed that basic residues within this IDR-2 domain (Figure 1) are essential to the process. Whether the C-terminus exerts phase separation condensates in living cells as well remains unclear, with the confounding issue that IDR-2 deletion removes the NID domain as well. Interestingly, however, an RTT-associated nonsense mutation that removed the entire IDR-2 gave strongly reduced accumulation of MeCP2 within heterochromatic foci. Concomitantly, reduced incorporation of a fluorophore-tagged $\mathrm{HP} 1 \alpha$ was observed within heterochromatin in these mutant cells. In vitro, concordantly, phase-separated MeCP2 droplets incorporated tagged-HP1 $\alpha$ when this was added to the tube, showing that these two proteins can form joint condensates [6]. These observations are interesting given that also HP1 $\alpha$ displays phase separation properties - at least in vitro - and given that this heterochromatin component restricts the compaction levels of heterochromatin in somatic cells [16,17].

Further studies are required to unravel the molecular details, for instance of how MeCP2 and the HP1 isoform $\mathrm{HP} 1 \alpha$ are linked within heterochromatin. The data so far evoke a model in which local methyl-CpG (and methyl$\mathrm{CAC}$ ) concentration triggers the initial $\mathrm{MeCP} 2$ enrichment at heterochromatin, which requires the MBD domain (Figure 1). Further protein condensation is achieved through the LLPS-like properties of the IDR-2. The phase separation of MeCP2, HP1 $\alpha$ and yet other heterochromatin components may be facilitated by the focally high concentration of nucleosomes as well [18]. In neurons, MeCP2 condensation within heterochromatin correlates with focal HP1 $\alpha$ enrichment, reduced H4K20me3 and with lower compaction levels (Figure 1). Whether MeCP2 condensation also influences HP1 $\beta$ levels would now be interesting to explore, given that this HP1 isoform is functionally associated with H4K20me3 and with increased chromatin compaction levels [16].

What could be the role of the intriguing MeCP2-mediated heterochromatin configuration? Without the MBDinduced recruitment of MeCP2 to its methylated DNA, a further accumulation - possibly through LLPS - would not take place at heterochromatin [6]. Molecular details are yet insufficient, however, to understand the dampening effects of $\mathrm{MeCP} 2$ condensates on repressive histone methylation and compaction levels.

A missing piece of the puzzle could be RNA. In recent studies, different nuclear RNAs were reported to influence chromatin structure and the formation of protein condensates in distinct nuclear compartments [19], including at pericentromeric heterochromatin [20]. Within this context, Fioriniello et al. recently explored the importance of RNA for heterochromatic MeCP2 condensates in neural cells [5]. After a brief RNase-A treatment, which completely ablated the major satellite RNA, strongly-reduced levels of MeCP2 were observed at heterochromatin foci. A similar, but smaller, effect was seen in experiments in which the authors used RNA-ablating gapmers directed against satellite repeat RNA [5]. In Mecp2 KO cells, conversely, major-satellite RNAs (transcribed in the forward orientation) were strongly reduced at heterochromatin. Dissecting this further in missense mutations, the authors found that the MBD and the NID-comprising transcriptional repression domain are both required for the targeting of satellite-RNA to heterochromatin. Combined with additional experiments, the authors propose that MeCP2 interacts with major-satellite RNAs and contributes to their retention at heterochromatin. The major-satellite repeat RNAs, in turn, contribute to focal MeCP2 condensation, as they do for other heterochromatin components [20,21], and this could involve dose-dependent threshold effects.

The recent studies generated intriguing insights into $\mathrm{MeCP} 2$ and heterochromatin, but also evoke new questions. Notably, it remains unclear how important the structural effects of MeCP2 on heterochromatin are in relation to RTT. Several of the mutations that had chromatin-structural effects are causally involved in RTT [6], but this might be for other reasons not related to heterochromatin, including possible effects on MBD or NID functions or on the protein's stability. In Mecp2 KO mice, the different RTT-like neurological defects can be prevented or reversed by expressing a truncated mini-protein that comprises just the MBD and NID domains [22]. This suggests that the neuronal functions mediated by $\mathrm{MeCP} 2$ are possibly not linked to the protein's structural effects on heterochromatin, in case the latter are indeed mediated by regions other than the MBD and NID [6].

Irrespective of the precise underlying mechanisms, the novel studies clearly establish a role for MeCP2 in the architecture and compaction of heterochromatin, and reveal likely cooperation with HP1 proteins and majorsatellite RNAs. Future research will undoubtedly provide other pieces of this rather complicated puzzle.

\section{Acknowledgments}

We apologize to colleagues whose work was not cited due to space limitations. We thank D. Llères for expert advice and proofreading. 


\section{Financial \& competing interests disclosure}

Research in the R Feil laboratory is supported by the Agence Nationale de la Recherche (ANR), through grant ANR-18-CE12-0022-02 ('IMP-REGULOME') and Labex EpiGenMed (ANR-10-LABX-12-01), and the Institut National de Cancer (INCa, grant PLBIO18-094). Y Imaizumi acknowledges Fellowship funding from TOYOBO Biotechnology Foundation, Japan (2019-01). The authors have no relevant affiliations or financial involvement with any organization or entity with a financial interest in or financial conflict with the subject matter or materials discussed in the manuscript.

No writing assistance was utilized in the production of this manuscript.

\section{Open access}

This work is licensed under the Attribution-NonCommercial-NoDerivatives 4.0 Unported License. To view a copy of this license, visit http://creativecommons.org/licenses/by-nc-nd/4.0/

\section{References}

1. Hendrich B, Tweedie S. The methyl-CpG binding domain and the evolving role of DNA methylation in animals. Trends Genet. 19(5), 269-277 (2003).

2. Rausch C, Hastert FD, Cardoso MC et al. DNA modification readers and writers and their interplay. J. Mol. Biol. 432(6), 1731-1746 (2020).

3. Ito-Ishida A, Baker SA, Sillitoe RV. MeCP2 levels regulate the 3D structure of heterochromatic foci in mouse neurons. J. Neurosci. 40(45), 8746-8766 (2020).

4. Linhoff MW,, Garg SK, Mandel G. A high-resolution imaging approach to investigate chromatin architecture in complex tissues. Cell 163(1), 246-255 (2015).

5. Fioriniello $\mathrm{S}$, Csukonyi E, Marano $\mathrm{D}$ et al. MeCP2 and major satellite forward RNA cooperate for pericentric heterochromatin organization. Stem Cell Rep. 15(6), 1317-1332 (2020).

6. Li CH, Coffey EL, Dall'Agnese A et al. MeCP2 links heterochromatin condensates and neurodevelopmental disease. Nature 586(7829), 440-444 (2020).

7. Tillotson R, Bird A. The molecular basis of MeCP2 function in the brain. J. Mol. Biol. 432(6), 1602-1623 (2020).

8. Lombardi LM, Baker SA, Zoghbi HY , ,et al.,, MECP2 disorders: from the clinic to mice and back. J. Clin. Invest. 125(8), 2914-2923 (2015).

9. Guy J,Hendrich B, Holmes M, Martin JE, Bird A, A mouse Mecp2-null mutation causes neurological symptoms that mimic Rett syndrome. Nat. Genet. 27(3), 322-326 (2001).

10. Guy J,Gan J, Selfridge J, Cobb S, Bird A,, Reversal of neurological defects in a mouse model of Rett syndrome. Science 315(5815), 1143-1147 (2007).

11. Giacometti E,Luikenhuis S, Beard C, Jaenisch R,, Partial rescue of MeCP2 deficiency by postnatal activation of MeCP2. Proc. Nat. Acad. Sci. USA 104(6), 1931-1936 (2007).

12. Lagger $\mathrm{S}$, , Connelly JC, Schweikert G et al. MeCP2 recognizes cytosine methylated tri-nucleotide and di-nucleotide sequences to tune transcription in the mammalian brain. PLoS Genet. 13(5), e1006793 (2017).

13. Skene PJ, Illingworth RS, Webb $\mathrm{S}$ et al. Neuronal MeCP2 is expressed at near histone-octamer levels and globally alters the chromatin state. Mol.Cell 37(4), 457-468 (2010).

14. Chhatbar K,Cholewa-Waclaw J, Shah R, Bird A, Sanguinetti G,, Quantitative analysis questions the role of MeCP2 as a global regulator of alternative splicing. PLoS Genet. 16(10), e1009087 (2020).

15. Shoaib M, Walter D, Gillespie PJ et al. Histone H4K20 methylation mediated chromatin compaction threshold ensures genome integrity by limiting DNA replication licensing. Nat.Commun. 9, 3704 (2018).

16. Bosch-Presegue L, Raurell-Vila H, Thackray JK et al. Mammalian HP1 isoforms have specific roles in heterochromatin structure and organization. Cell Rep. 21(8), 2048-2057 (2017).

17. Sanulli S, Narlikar GJ. Liquid-like interactions in heterochromatin: implications for mechanism and regulation. Curr. Opin. Cell Biol. 64, 90-96 (2020).

18. Strickfaden H,Tolsma TO, Sharma A, Underhill DA, Hansen JC, Hendzel MJ,, Condensed chromatin behaves like a solid on the mesoscale in vitro and in living cells. Cell 183(7), 1772-1784.e13 (2020).

19. Thakur J, Henikoff S. Architectural RNA in chromatin organization. Biochem. Soc. Trans. 48(5), 1967-1978 (2020).

20. Huo XR, Ji LZ, Zhang YW, Lv P et al. The nuclear matrix protein SAFB cooperates with major satellite rnas to stabilize heterochromatin architecture partially through phase separation. Mol. Cell 77(2), 368-383.e7 (2020).

21. Camacho OV, Galan C, Swist-Rosowska K et al. Major satellite repeat RNA stabilize heterochromatin retention of Suv39h enzymes by RNA-nucleosome association and RNA:DNA hybrid formation. eLife 6, e25293 (2017). 
22. Tillotson R, Selfridge J, Koerner MV et al. Radically truncated MeCP2 rescues Rett syndrome like neurological defects. Nature 550(7676), 398-401 (2017). 
